\title{
Patient Reported Outcomes (PROs) as Part of Value- Based Care Can Shape Therapy Guidelines: Impact on Emerging Targeted Agents and Immunotherapy Protocols in Resource-Limited Regions
}

Frank E. Mott (i)

Received: October 25, 2016/Published online: January 5, 2017

(C) The Author(s) 2017. This article is published with open access at Springerlink.com

\begin{abstract}
Patient reported outcomes (PROs) are commonly integrated into clinical trials for new cancer therapies or treatment modalities; however, beyond a cursory review during the registration process for a new drug, these important measures of patient satisfaction and cost-effectiveness are often under-used in daily practice. We cite literature under-scoring the value of PROs not only in clinical trials, but in practical decision making and how such metrics can help guide the oncologist and the patient in choosing the best and most cost-effective therapy for their cancer. This is especially critical with the advent of new immunotherapies that are proving to be therapeutically beneficial for many patients, but not all, and at substantial cost in terms of toxicities and financial impact.
\end{abstract}

Enhanced content To view enhanced content for this article go to http://www.medengine.com/Redeem/ D157F0601DEB9DE2.

F. E. Mott $(\bowtie)$

Department of Thoracic Head Neck Medical

Oncology, MD Anderson Cancer Center, Houston,

TX, USA

e-mail: fmott@mdanderson.org
Keywords: Cost effectiveness; Immunotherapy; Patient outcomes; Resources; Targeted therapy

The past decade has seen remarkable progress in cancer treatment. Much of this has been due to the improvements in the ability to identify potentially targetable biomarkers. The identification of genetic aberrations has led to new therapies for lung cancer that are now achieving response rates approaching 90\% in select patient subsets with tumors that are enriched for the specific target [1]. Better understanding of the pathways involved in immune tolerance and rejection have led to development of therapies to modulate those pathways and have turned previously untreatable cancers into chronic diseases.

After a slow start, we are now seeing an explosive growth in development of new drugs in these areas. In 2015 alone, there were no less than 32 approvals by the Food and Drug Administration (FDA) for new agents or new indications for existing agents in the treatment of various cancers [2]. The FDA has already granted 12 approvals for the first half of 2016. While this rapid expansion offers new therapeutic options for patients where they 
did not exist before, it does not come without a cost.

Several of the recent FDA approvals have been for "me-too" drugs, agents that use a similar mechanism of action and with similar toxicity profiles as agents already on the market. For example, nivolumab, an antibody that targets the Programmed Death (PD-1) receptor, which had already demonstrated activity in metastatic melanoma, was first approved in March 2015 for second-line therapy of advanced squamous non-small cell lung cancer (NSCLC) in patients progressing on or after prior platinum-based chemotherapy [3, 4]. In October, 2015, the FDA expanded nivolumab's indication to include NSCLCs of the adenocarcinoma variety as well $[5,6]$. That same month, the FDA granted approval for pembrolizumab as second-line therapy in platinum-treated NSCLC patients, but with the requirement that they demonstrated positive Program Death Ligand 1 (PD-L1) expression $[7,8]$. Currently there are a number of additional PD-1/PD-L1 inhibitors going through clinical trials for NSCLC and other solid tumors.

These agents also have shown activity in other cancers, leading to expanded FDA approvals for melanoma, renal cell carcinoma, and bladder urothelial cancers among others. Other targeted agents are now available or being studied for hematologic malignancies, breast cancers, head and neck cancers, brain metastases, and others. While some of this rapid therapeutic expansion is driven by a need to improve existing treatment options, marketing and pharmaceutical patents also play a role as companies jockey to be the first to gain FDA-approval for their particular drug.

The cost of these new therapies can be staggering. A Wall Street Journal report in 2015 estimated the cost of nivolumab and ipilimumab for metastatic melanoma at $\$ 141,000$ for the first 12 weeks and $\$ 256,000$ for the first year of therapy [9]. Nivolumab monotherapy is approved for both squamous and non-squamous NSCLC as second-line therapy after prior platinum-based therapy, as noted earlier. This is based on the CheckMate trials comparing nivolumab versus docetaxel $[4,6]$. Several countries have tried to analyze the cost-effectiveness of nivolumab in this setting. A United Kingdom study in 2015 reported that nivolumab in squamous NSCLC was not cost-effective per quality-adjusted life years (QALY) gained, and a similar Canadian study this year comparing nivolumab with docetaxel and erlotinib in NSCLC showed nivolumab with the highest per-patient cost $[10,11]$. A more recent Swiss study looking at nivolumab versus docetaxel in non-squamous NSCLC using clinical data from the CheckMate-057 trial measured incremental cost effectiveness ratios (ICER) per QALY-gained [12]. The study showed that for all patients treated, nivolumab had much higher ICERs (in Swiss francs) than docetaxel; however, for patients with positive PD-L1 expression $[>1 \%$ or $>10 \%$ by immunohistochemical (IHC) staining], the nivolumab ICERs were lower and felt to be cost-effective based on a willingness-to-pay (WTP) threshold specific for the Swiss health-care system. Thus, the authors felt that for nivolumab to be cost-effective either the drug price and/or dose needed to be reduced or treatment based on PD-L1 testing should be considered.

Even for patients with good insurance coverage, this can be a daunting price tag. The economics of health care is complex and beyond the scope of this editorial. Health care policy is a moving target, but one that occupies a major component of our federal budget. Reimbursement for medical care is moving 
towards value-based outcomes, rather than procedural or volume-based plans [13]. Providers will not be paid for simply seeing more patients, ordering more tests, and delivering more treatments. The quality of those interactions, including patient outcomes, will be important in determining value-based reimbursement levels.

With the plethora of agents emerging, clinicians are confronted with challenging choices in determining the best treatment option for specific patients. Patients are also confronted with decisions related to the procedures involved in diagnosis to the potential side effects and costs of new therapies. Insurance coverage and access to centers familiar with these procedures and treatments present potential barriers for many patients. It is in this climate that patient reported outcomes (PROs) are increasingly important as we try to determine the best value of care and use of these new technologies and treatment options [14, 15].

In 2013, the National Quality Forum (NQF), an organization that reviews and endorses quality metrics published the conclusions of an expert panel charged with developing standards for metrics in PRO development [16]. Their work was endorsed by the International Society for Quality of Life Research [17]. Their six-step pathway consisted of the following: identify performance concerns for the population of interest; determine meaningful outcomes; develop PROs to measure those outcomes; determine PRO-performance measures (PRO-PMs); conduct pilot testing; standardize guidelines. PROs have already demonstrated impact on value care decision making. The American Society of Clinical Oncology (ASCO) used the NQF model to pilot specific oncology programs for pain assessment in patients with bone metastases and post-chemotherapy nausea control [14]. For patients with cancer-related pain, the work group established that the Brief Pain Inventory (BPI) was the most appropriate tool for PRO measurement of pain [18]. Their consensus was that a PRO Performance Measure should assess the proportion of patients with metastatic disease with a worst pain score of $\geq 4$ as a group that would likely benefit from modification of their analgesic regimens. Similarly, for nausea due to moderately or highly emetogenic chemotherapy, their consensus developed a PRO-PM for patients who develop nausea within the first week after receiving such chemotherapy and to rate their worst nausea and frequency of nausea with potential interventions to modify the anti-emetic regimens for such patients.

These are two examples where PRO-PMs are helpful in modifying treatment algorithms to achieve value based and patient preferred therapy. There are many areas in cancer treatment that could benefit from PRO-PMs incorporated into the treatment strategy or as part of an ongoing clinical trial. As an example, identifying targetable mutations or gene re-arrangements currently requires adequate tissue samples, often necessitating invasive and painful biopsy procedures, sometimes more than once. The risk and cost of these procedures is not insignificant and patient compliance can be negatively impacted. Research is ongoing to develop liquid biopsies, circulating free tumor DNA, which may obviate the need for invasive surgical biopsies in many cases $[19,20]$. Patients uniformly say they would prefer and be more compliant with liquid biopsies as an alternative to tissue sampling if the accuracy of such testing was as good [21].

Similarly, PRO assessment of quality of life metrics and toxicities can help define a value-based choice of treatment for a particular 
cancer. Coming to clinic for treatment once every 3 weeks versus every 2 weeks may be an important variable for patients still trying to balance a family or work life with their treatment. Co-pays and provider costs for the patient, not covered by their insurance, may be better absorbed with less frequent treatment visits. If toxicities on the two treatments are otherwise identical and the efficacy comparable, then patients may be better served with a 3-week frequency. This is an example of PRO influencing value based care decisions.

These factors create an imperative that we look at our new therapeutic and diagnostic advances not simply as clinical breakthroughs, but also in the context of their impact on health care economics and patient outcomes, including quality and quantity of life and at what cost, both physically and financially. While response rates, progression free and overall survival, and toxicity grades are important, including PRO surveys and other mechanisms as part of the clinical trial could provide valuable insight into the patient's perspective and the treatments' impact on their life. These added metrics, combined with clinical outcomes, could help determine the best value approach for a particular diagnosis and a patient centered care that would be cost effective as well.

The previously described ASCO workgroup's concluding statements that "patient self-reporting affords the opportunity to understand better the impact of care processes on how patients feel (and can optimize) good clinical practice" is indicative of how important this is to patients. As such, it is important to incorporate such metrics into the evaluation of new therapies in order to provide quality effective care along with quality of life and control of cost. ASCO has lobbied for incorporating PROs into clinical practice. As such, it may be time to standardize PRO surveys.
The six-step approach outlined by the NQF may be one example. Validation of surveys is important. At MD Anderson Cancer Center, we have begun incorporating the MD Anderson Symptom Inventory (MDASI), a brief multi-symptom questionnaire, as a scale for outcomes evaluation of patients on various immunotherapy trials [22]. With the consensus recommendations of organizations such as ASCO, the NQF, and the International Society for Quality of Life Research, amongst others, might it be timely to incorporate routinely PRO-PMs and PRO surveys in clinical trials and registration studies as part of the approval process for new treatment paradigms and/or individual drugs? The future economic health of cancer care may depend on it.

\section{ACKNOWLEDGEMENTS}

No funding or sponsorship was received for this study or publication of this article. All named authors meet the International Committee of Medical Journal Editors (ICMJE) criteria for authorship for this manuscript, take responsibility for the integrity of the work as a whole, and have given final approval for the version to be published.

Disclosures. Frank E. Mott has nothing to disclose.

Compliance with Ethics Guidelines. This article is based on previously conducted studies and does not involve any new studies of human or animal subjects performed by any of the authors.

Data Availability. Data sharing is not applicable to this article as no datasets were generated or analyzed during the current study. 
Open Access. This article is distributed under the terms of the Creative Commons Attribution-NonCommercial 4.0 International License (http://creativecommons.org/licenses/ by-nc/4.0/), which permits any noncommercial use, distribution, and reproduction in any medium, provided you give appropriate credit to the original author(s) and the source, provide a link to the Creative Commons license, and indicate if changes were made.

\section{REFERENCES}

1. Seto T, Kiura K, Nishio M, et al. CH5424802 (RO5424802) for patients with ALK-rearranged advanced non-small-cell lung cancer (AF-001JP study): a single-arm, open-label, phase 1-2 study. Lancet Oncol. 2013;14:590-8.

2. Hematology/Oncology (Cancer) Approvals and Safety Notifications. US Food and Drug Administration. http://www.fda.gov.

3. Nivolumab (Opdivo) FDA approval-US Food and Drug Administration; March 4, 2015. http://www. fda.gov/drugs/informationondrugs/approveddrugs/ ucm436566.htm.

4. Brahmer J, Reckamp KL, Baas P, et al. Nivolumab versus docetaxel in advanced squamous cell non-small cell lung cancer. $\mathrm{N}$ Engl $\mathrm{J}$ Med. 2015;373:123-35.

5. Nivolumab (Opdivo) FDA approval-US Food and Drug Administration; October 9, 2015. http://www. fda.gov/drugs/informationondrugs/approveddrugs/ ucm466576.htm

6. Borghaei H, Pax-Ares L, Horn L, et al. Nivolumab versus docetaxel in advanced non-squamous non-small cell lung cancer. $N$ Engl J Med. 2015;373:1627-39.

7. Pembrolizumab (Keytruda) FDA approval-US Food and Drug Administration; October 2, 2015. http:// www.fda.gov/drugs/informationondrugs/approved drugs/ucm465650.htm.

8. Garon EB, Rizvi NA, Hui R, et al. Pembrolizumab for the treatment of non-small-cell lung cancer. N Engl J Med. 2015;372:2018-28.
9. Loftus P, Winslow R. FDA approves Bristol-Myers's Yervoy, Opdivo for treatment of melanoma. The Wall Street Journal. October 1, 2015.

10. National Institute for Health and Care Excellence. Nivolumab for previously treated locally advanced or metastatic squamous non-small cell lung cancer. https://s3.amazonaws.com/assets.fiercemarkets.net/ public/005-LifeSciences/niceopdivoappraisal.pdf. Accessed Feb 292016.

11. Goeree R, Villeneuve J, Goeree J, et al. Economic evaluation of nivolumab for the treatment of second-line advanced squamous NSCLC in Canada: a comparison of modelling approaches to estimate and extrapolate survival outcomes. J Med Econ. 2016;19(6):630-44.

12. Matter-Walstra K, Schwenkglenks M, Aebi S, et al. A cost-effectiveness analysis of nivolumab versus docetaxel for advanced non-squamous NSCLC including PD-L1 testing. J Thoracic Oncol. 2016. http://www.jto.org/article/S1556-0864(16)30507-X/ abstract.

13. Centers for Medicare and Medicaid Services Value-Based Programs. CMS.Gov. https://www. cms.gov/Medicare/Quality-Initiatives-Patient-Asse ssment-Instruments/Value-Based-Programs/ValueBased-Programs.html.

14. Basch E, Snyder C, McNiff K, et al. Patient-reported outcome performance measures in Oncology. J Oncol Prac. 2014;10(3):209-11.

15. Gnanasakthy A, DeMuro C, Clark $M$, et al. Patient-reported outcomes labeling for products approved by the office of hematology and oncology products of the US Food and Drug Administration (2010-2014). J Clin Oncol. 2016;34:1928-34.

16. National Quality Forum: patient-reported outcomes in performance measurement. Washington, DC, National Quality Forum, January 10, 2013. http:// www.qualityforum.org/Projects/n-r/Patient-Reported_ Outcomes/Patient-Reported_Outcomes.aspx.

17. Basch E, Wu A, Moinpour CM, et al. Steps for assuring rigor and adequate patient representation when using patient-reported outcome performance measures (PRO-PMs). Plymouth Meeting, PA, National Quality Measures Clearinghouse, 2013, pp 1-5.

18. Shi Q, Wang XS, Mendoza TR, et al. Assessing persistent cancer pain: a comparison of current pain ratings and pain recalled from the past week. J Pain Symptom Manage. 2009;37:168-74. 
19. Heitzer E, Ulz P, Geig J. Circulating tumor DNA as a liquid biopsy for cancer. Clin Chem. 2015;61:112-23.

20. Diaz L, Bardelli A. Liquid biopsies: genotyping circulating tumor DNA. J Clin Oncol. 2014;32:579-86.

21. Zill O, Mortimer S, Banks K, et al. Somatic genomic landscape of over 15,000 patients with advanced-stage cancer from clinical next-generation sequencing analysis of circulating tumor DNA. ASCO 2016. J Clin Oncol 2016;34:suppl; abstr LBA11501.

22. Cleeland CS, Mendoza TR, Wang XS, et al. Assessing symptom distress in cancer patients: the M.D. Anderson Symptom Inventory. Cancer. 2000;89(7):1634-46. 